\title{
Self-Similar Solutions of the Non-Strictly Hyperbolic Whitham Equations for the KdV Hierarchy
}

\author{
V. U. Pierce and Fei-Ran Tian \\ Communicated by Y. Charles Li, received April 18, $200 \%$.
}

\begin{abstract}
We study the Whitham equations for all the higher order KdV equations. The Whitham equations are neither strictly hyperbolic nor genuinely nonlinear. We are interested in the solution of the Whitham equations when the initial values are given by a step function.
\end{abstract}

\section{Contents}

1. Introduction 263

2. The Whitham Equations 266

3. Self-similar Solutions 270

4. The Minimization Problem 277

$\begin{array}{ll}\text { References } & 281\end{array}$

\section{Introduction}

It is known that the solution of the $\mathrm{KdV}$ equation

$$
u_{t}+6 u u_{x}+\epsilon^{2} u_{x x x}=0
$$

has a weak limit as $\epsilon \rightarrow 0$ while the initial values

$$
u(x, 0 ; \epsilon)=u_{0}(x)
$$

are fixed.

This weak limit is described by hyperbolic equations. It satisfies the Burgers equation

$$
u_{t}+\left(3 u^{2}\right)_{x}=0
$$

1991 Mathematics Subject Classification. 35, 76.

Key words and phrases. Whitham equations, KdV equation, Self-Similar Solution, KdV Hierarchy. 
until its solution develops shocks. Immediately after shock, the weak limit is governed by the Whitham equations $[\mathbf{5}, \mathbf{6}, \mathbf{1 4}, \mathbf{1 5}]$

$$
u_{i t}+\lambda_{i}\left(u_{1}, u_{2}, u_{3}\right) u_{i x}=0, \quad i=1,2,3,
$$

where the $\lambda_{i}$ 's are given by formulae (2.12). Equations (1.3) form a $3 \times 3$ system of hyperbolic equations. After the breaking of the solution of (1.3), the weak limit is described by a $5 \times 5$ systems of hyperbolic equations similar to (1.3). Similarly, after the solution of the $5 \times 5$ system breaks down, the weak limit is characterized by a $7 \times 7$ system of hyperbolic equations. In other words, for general initial data $u_{0}(x)$, one constructs the weak limit by patching together solutions of (1.2), (1.3), $5 \times 5,7 \times 7$, etc systems in the $x$ - $t$ plane.

The KdV equation (1.1) is just the first of an infinite sequence of equations. All these so-called higher order KdV equations can be cast in the Hamiltonian form

$$
\frac{\partial u}{\partial t}+\frac{\partial}{\partial x} \frac{\delta H_{m}}{\delta u}=0, \quad m=1,2, \cdots,
$$

where $H_{m}$ 's form a sequence of conserved functionals for the KdV equation. The small dispersive parameter $\epsilon$ is hidden in $H_{m}$. In particular, when $m=1,(1.4)$ is the $\mathrm{KdV}$ equation.

The solution of the higher order KdV equation (1.4) also has a weak limit as $\epsilon \rightarrow 0$. As in the $\mathrm{KdV}$ case, this weak limit satisfies the Burgers type equation

$$
u_{t}+\left(\frac{C_{m}}{m+1} u^{m+1}\right)_{x}=0,
$$

where $C_{m}$ is given in (2.20), until the solution of (1.5) forms a shock. After the breaking of the solution of (1.5), the limit is governed by equations similar to (1.3), namely,

$$
u_{i t}+\mu_{i}^{(m)}\left(u_{1}, u_{2}, u_{3}\right) u_{i x}=0, \quad i=1,2,3,
$$

where $\mu_{i}^{(m)}$ 's are given in (2.15). They will also be called the Whitham equations. As in the KdV case, after the solution of (1.6) breaks down, the weak limit is described by a $5 \times 5$ system of hyperbolic equations.

In this paper, we are interested in the solution of the Whitham equations for the higher order $\mathrm{KdV}(1.4)$ with a step-like initial function

$$
u_{0}(x)=\left\{\begin{array}{cc}
1 & x<0 \\
0 & x>0
\end{array}\right.
$$

For such an initial function, the solution of the Burgers type equation (1.5) has already developed a shock at the initial time, $t=0$. Hence, immediately after $t=0$, the Whitham equations (1.6) kick in. Solutions of (1.6) occupy some domains of the space-time while solutions of (1.5) occupy other domains. These solutions are matched on the boundaries of the domains.

Equations (1.2) and equations (1.5) are prototypes in the theory of hyperbolic conservation laws $[\mathbf{7}]$. Their solutions will generally develop shocks in finite times. The solutions can be extended beyond the singularities as the entropy solutions.

Solutions of equations (1.2) or equations (1.5), in the theory of the zero dispersion limit, are not extended as weak or entropy solutions after the formation of singularities. Instead, they are extended to match the Whitham solutions of (1.3) or (1.6). For initial data (1.7), the resulting solutions of the Whitham equations (1.6) will be seen to be more complex than those of (1.3) in the KdV case. 
The KdV case with the step-like initial data (1.7) was first studied by Gurevich and Pitaevskii [3]. They found that it was enough to use the Burgers solution of (1.2) and the Whitham solution of (1.3) to cover the whole $x$ - $t$ plane, without going to the $5 \times 5$ or $7 \times 7$ system. Namely, the space-time is divided into three parts

$$
\text { (1) } \frac{x}{t}<-6, \quad \text { (2) }-6<\frac{x}{t}<4, \quad \text { (3) } \frac{x}{t}>4 \text {. }
$$

The solution of (1.2) occupies the first and third parts,

$$
u(x, t) \equiv 1 \quad \text { when } \frac{x}{t}<-6, \quad u(x, t) \equiv 0 \quad \text { when } \frac{x}{t}>4
$$

The Whitham solution of (1.3) lives in the second part,

$$
u_{1}(x, t) \equiv 1, \quad \frac{x}{t}=\lambda_{2}\left(1, u_{2}, 0\right), \quad u_{3}(x, t) \equiv 0,
$$

when $-6<x / t<4$.

Whether the second equation of (1.9) can be inverted to give $u_{2}$ as a function of the self-similarity variable $x / t$ hinges on whether

$$
\frac{\partial \lambda_{2}}{\partial u_{2}}\left(1, u_{2}, 0\right) \neq 0 .
$$

Indeed, Levermore [8] has proved the genuine nonlinearity of the Whitham equations (1.3), i.e.,

$$
\frac{\partial \lambda_{i}}{\partial u_{i}}\left(u_{1}, u_{2}, u_{3}\right)>0, \quad i=1,2,3
$$

for $u_{1}>u_{2}>u_{3}$.

For the higher order $\mathrm{KdV}$ (1.4), equations (1.6), in general, are not genuinely nonlinear, i.e., a property like (1.10) is not available. Hence, solutions like (1.8) and (1.9) need to be modified.

Our construction of solutions of the Whitham equation (1.6) makes use of the non-strict hyperbolicity of the equations. For KdV, it is known that the Whitham equations (1.3) are strictly hyperbolic, namely:

$$
\lambda_{1}\left(u_{1}, u_{2}, u_{3}\right)>\lambda_{2}\left(u_{1}, u_{2}, u_{3}\right)>\lambda_{3}\left(u_{1}, u_{2}, u_{3}\right)
$$

for $u_{1}>u_{2}>u_{3}[8]$. For the higher order $\operatorname{KdV}(1.4)$, different eigenspeeds of (1.6), $\mu_{i}^{(m)}\left(u_{1}, u_{2}, u_{3}\right)$ 's, may coalesce in the region $u_{1}>u_{2}>u_{3}[\mathbf{9}]$.

For the higher order $\mathrm{KdV}$ with step-like initial function (1.7), the space time is divided into four regions (see Figure 1.)

$$
\text { (1) } \frac{x}{t}<\alpha, \quad \text { (2) } \alpha<\frac{x}{t}<\beta, \quad \text { (3) } \beta<\frac{x}{t}<4^{m}, \quad \text { (4) } \frac{x}{t}>4^{m},
$$

where $\alpha$ and $\beta$ are some constants. In the first and fourth regions, the solution of (1.5) governs the evolution:

$$
u(x, t) \equiv 1 \quad \text { where } x / t<\alpha \text { and } u(x, t) \equiv 0 \quad \text { where } x / t>4^{m} .
$$

The Whitham solution of (1.6) lives in the second and third regions; namely:

$$
u_{1}(x, t) \equiv 1, \quad \frac{x}{t}=\mu_{2}^{(m)}\left(1, u_{2}, u_{3}\right), \quad \frac{x}{t}=\mu_{3}^{(m)}\left(1, u_{2}, u_{3}\right),
$$

when $\alpha<x / t<\beta$, and

$$
u_{1}(x, t) \equiv 1, \quad \frac{x}{t}=\mu_{2}^{(m)}\left(1, u_{2}, 0\right), \quad u_{3}(x, t) \equiv 0,
$$

when $\beta<x / t<4^{m}$. 
Equations (1.11) yield

$$
\mu_{2}^{(m)}\left(1, u_{2}, u_{3}\right)=\mu_{3}^{(m)}\left(1, u_{2}, u_{3}\right)
$$

on a curve in the region $0<u_{3}<u_{2}<1$. This implies the non-strict hyperbolicity of the Whitham equations (1.6) for the KdV hierarchy.

The $m=2$ case has been studied in $[\mathbf{9}]$. There, inequalities

$$
\frac{\partial \mu_{3}^{(m)}}{\partial u_{3}}<\frac{3}{2} \frac{\mu_{2}^{(m)}-\mu_{3}^{(m)}}{u_{2}-u_{3}}<\frac{\partial \mu_{2}^{(m)}}{\partial u_{2}} \text { for } u_{1}>u_{2}>u_{3}>0
$$

have played a crucial role in verifying that equations (1.11) or (1.12) can indeed be solved to give the solution of the Whitham equations (1.6) when $m=2$.

For $m>2$, inequalities (1.13) are not valid any more. We therefore must use a different approach to solve the problem. The calculations are considerably more difficult than in the $m=2$ case. This is mainly because $q$ of (2.25) is a polynomial of degree $m$ when $m>2$ while it is only a quadratic polynomial when $m=2$.

The organization of the paper is as follows. In Section 2, we will study the eigenspeeds, $\mu_{i}^{(m)}$ 's, of the Whitham equations (1.6). In Section 3, we will construct the self-similar solution of the Whitham equations for the initial function (1.7). In Section 4, we will use the self-similar solution of Section 3 to construct the minimizer of a variational problem for the zero dispersion limit of the KdV hierarchy.

In a subsequent publication, we will study the Whitham solutions for all the other step-like initial data.

\section{The Whitham Equations}

In this section we define the eigenspeeds of the Whitham equations for both the $\mathrm{KdV}$ (1.1) and higher order KdV (1.4). We first introduce the polynomials of $\xi$ for $n=0,1,2, \ldots[\mathbf{1}, \mathbf{4}, \mathbf{1 2}]$ :

$$
P_{n}\left(\xi, u_{1}, u_{2}, u_{3}\right)=\xi^{n+1}+a_{n, 1} \xi^{n}+\cdots+a_{n, n+1},
$$

where the coefficients, $a_{n, 1}, a_{n, 2}, \ldots, a_{n, n+1}$ are uniquely determined by the two conditions

$$
\frac{P_{n}\left(\xi, u_{1}, u_{2}, u_{3}\right)}{\sqrt{\left(\xi-u_{1}\right)\left(\xi-u_{2}\right)\left(\xi-u_{3}\right)}}=\xi^{n-1 / 2}+\mathcal{O}\left(\xi^{-3 / 2}\right) \text { for large }|\xi|
$$

and

$$
\int_{u_{3}}^{u_{2}} \frac{P_{n}\left(\xi, u_{1}, u_{2}, u_{3}\right)}{\sqrt{\left(\xi-u_{1}\right)\left(\xi-u_{2}\right)\left(\xi-u_{3}\right)}} d \xi=0 .
$$

Here the sign of the square root is given by $\sqrt{\left(\xi-u_{1}\right)\left(\xi-u_{2}\right)\left(\xi-u_{3}\right)}>0$ for $\xi>u_{1}$ and the branch cuts are along $\left(-\infty, u_{3}\right)$ and $\left(u_{2}, u_{1}\right)$.

In particular,

$$
P_{0}\left(\xi, u_{1}, u_{2}, u_{3}\right)=\xi+a_{0,1}, \quad P_{1}\left(\xi, u_{1}, u_{2}, u_{3}\right)=\xi^{2}-\frac{1}{2}\left(u_{1}+u_{2}+u_{3}\right) \xi+a_{1,2},
$$

where

$$
\begin{aligned}
& a_{0,1}=\left(u_{1}-u_{3}\right) \frac{E(s)}{K(s)}-u_{1}, \\
& a_{1,2}=\frac{1}{3}\left(u_{1} u_{2}+u_{1} u_{3}+u_{2} u_{3}\right)+\frac{1}{6}\left(u_{1}+u_{2}+u_{3}\right) a_{0,1} .
\end{aligned}
$$


Here

$$
s=\frac{u_{2}-u_{3}}{u_{1}-u_{3}}
$$

and $K(s)$ and $E(s)$ are complete elliptic integrals of the first and second kind.

$K(s)$ and $E(s)$ have some well-known properties $[\mathbf{1 0}, \mathbf{1 1}]$. They have the expansions

$$
\begin{aligned}
& K(s)=\frac{\pi}{2}\left[1+\frac{s}{4}+\frac{9}{64} s^{2}+\cdots+\left(\frac{1 \cdot 3 \cdots(2 n-1)}{2 \cdot 4 \cdots 2 n}\right)^{2} s^{n}+\cdots\right] \\
& E(s)=\frac{\pi}{2}\left[1-\frac{s}{4}-\frac{3}{64} s^{2}-\cdots-\frac{1}{2 n-1}\left(\frac{1 \cdot 3 \cdots(2 n-1)}{2 \cdot 4 \cdots 2 n}\right)^{2} s^{n}-\cdots\right]
\end{aligned}
$$

for $|s|<1$. They also have the asymptotics

$$
\begin{aligned}
K(s) & \approx \frac{1}{2} \log \frac{16}{1-s} \\
E(s) & \approx 1+\frac{1}{4}(1-s)\left[\log \frac{16}{1-s}-1\right]
\end{aligned}
$$

as $s$ is close to 1 . Furthermore,

$$
\begin{aligned}
\frac{d K(s)}{d s} & =\frac{E(s)-(1-s) K(s)}{2 s(1-s)} \\
\frac{d E(s)}{d s} & =\frac{E(s)-K(s)}{2 s} .
\end{aligned}
$$

It immediately follows from (2.5) and (2.6) that

$$
\frac{1}{1-\frac{s}{2}}<\frac{K(s)}{E(s)}<\frac{1-\frac{s}{2}}{1-s} \quad \text { for } 0<s<1 .
$$

The eigenspeeds of the Whitham equations (1.3) are defined in terms of $P_{0}$ and $P_{1}$ of $(2.4)$,

which give

$$
\lambda_{i}\left(u_{1}, u_{2}, u_{3}\right)=12 \frac{P_{1}\left(u_{i}, u_{1}, u_{2}, u_{3}\right)}{P_{0}\left(u_{i}, u_{1}, u_{2}, u_{3}\right)}, \quad i=1,2,3
$$

$$
\begin{aligned}
& \lambda_{1}\left(u_{1}, u_{2}, u_{3}\right)=2\left(u_{1}+u_{2}+u_{3}\right)+4\left(u_{1}-u_{2}\right) \frac{K(s)}{E(s)}, \\
& \lambda_{2}\left(u_{1}, u_{2}, u_{3}\right)=2\left(u_{1}+u_{2}+u_{3}\right)+4\left(u_{2}-u_{1}\right) \frac{s K(s)}{E(s)-(1-s) K(s)}, \\
& \lambda_{3}\left(u_{1}, u_{2}, u_{3}\right)=2\left(u_{1}+u_{2}+u_{3}\right)+4\left(u_{2}-u_{3}\right) \frac{K(s)}{E(s)-K(s)} .
\end{aligned}
$$

In view of (2.5-2.8), we find that $\lambda_{1}, \lambda_{2}$ and $\lambda_{3}$ have behavior:

(1) At $u_{2}=u_{3}$ :

$$
\begin{aligned}
& \lambda_{1}\left(u_{1}, u_{2}, u_{3}\right)=6 u_{1}, \\
& \lambda_{2}\left(u_{1}, u_{2}, u_{3}\right)=\lambda_{3}\left(u_{1}, u_{2}, u_{3}\right)=12 u_{3}-6 u_{1} .
\end{aligned}
$$

(2) At $u_{1}=u_{2}$ :

$$
\begin{aligned}
& \lambda_{1}\left(u_{1}, u_{2}, u_{3}\right)=\lambda_{2}\left(u_{1}, u_{2}, u_{3}\right)=4 u_{1}+2 u_{3} \\
& \lambda_{3}\left(u_{1}, u_{2}, u_{3}\right)=6 u_{3}
\end{aligned}
$$


The eigenspeeds of the Whitham equations (1.6) are

$$
\mu_{i}^{(m)}\left(u_{1}, u_{2}, u_{3}\right)=4^{m}(2 m+1) \frac{P_{m}\left(u_{i}, u_{1}, u_{2}, u_{3}\right)}{P_{0}\left(u_{i}, u_{1}, u_{2}, u_{3}\right)}, \quad i=1,2,3 .
$$

The polynomial $4^{m}(2 m+1) P_{m}\left(\xi, u_{1}, u_{2}, u_{3}\right)$ can be expressed as [2]

$$
\begin{aligned}
& 4^{m}(2 m+1) P_{m}\left(\xi, u_{1}, u_{2}, u_{3}\right)= \\
& 2\left(\xi-u_{1}\right)\left(\xi-u_{2}\right)\left(\xi-u_{3}\right) \Phi\left(\xi, u_{1}, u_{2}, u_{3}\right)+Q\left(\xi, u_{1}, u_{2}, u_{3}\right) .
\end{aligned}
$$

The function $\Phi(\xi, \vec{u})$ satisfies the boundary value problem for the Euler-PoissonDarboux equations

$$
\begin{aligned}
2\left(u_{i}-u_{j}\right) \frac{\partial^{2} \Phi}{\partial u_{i} \partial u_{j}} & =\frac{\partial \Phi}{\partial u_{i}}-\frac{\partial \Phi}{\partial u_{j}}, \\
2\left(\xi-u_{i}\right) \frac{\partial^{2} \Phi}{\partial \xi \partial u_{i}} & =\frac{\partial \Phi}{\partial \xi}-2 \frac{\partial \Phi}{\partial u_{i}} \\
\Phi(u, u, u, u) & =\frac{2}{3} \frac{d^{2}}{d u^{2}}\left[C_{m} u^{m}\right]
\end{aligned}
$$

where

$$
C_{m}=\frac{2^{2 m+1}}{\int_{0}^{1} \frac{t^{m}}{\sqrt{1-t}} d t}=\frac{2^{m}(2 m+1) ! !}{m !} .
$$

The function $Q(\xi, \vec{u})$ is a quadratic polynomial in $\xi$;

$$
\begin{aligned}
& Q\left(\xi, u_{1}, u_{2}, u_{3}\right)=2\left(\xi-u_{2}\right)\left(\xi-u_{3}\right) \frac{\partial q\left(u_{1}, u_{2}, u_{3}\right)}{\partial u_{1}} \\
& +2\left(\xi-u_{1}\right)\left(\xi-u_{3}\right) \frac{\partial q\left(u_{1}, u_{2}, u_{3}\right)}{\partial u_{2}}+2\left(\xi-u_{1}\right)\left(\xi-u_{2}\right) \frac{\partial q\left(u_{1}, u_{2}, u_{3}\right)}{\partial u_{3}} \\
& +q\left(u_{1}, u_{2}, u_{3}\right) P_{0}\left(\xi, u_{1}, u_{2}, u_{3}\right)
\end{aligned}
$$

and $q(\vec{u})$ is the solution of the boundary value problem for another version of the Euler-Poisson-Darboux equations

$$
\begin{aligned}
2\left(u_{i}-u_{j}\right) \frac{\partial^{2} q}{\partial u_{i} \partial u_{j}} & =\frac{\partial q}{\partial u_{i}}-\frac{\partial q}{\partial u_{j}}, \quad i, j=1,2,3, \\
q(u, u, u) & =C_{m} u^{m} .
\end{aligned}
$$

The solution of equations (2.17-2.19) and that of (2.22) and (2.23) can be solved explicitly [2]. In particular, the solution of (2.22) and (2.23) is [10]

$$
q\left(u_{1}, u_{2}, u_{3}\right)=\frac{C_{m}}{2 \sqrt{2} \pi} \int_{-1}^{1} \int_{-1}^{1} \frac{\left(\frac{1+\mu}{2} \frac{1+\nu}{2} u_{1}+\frac{1+\mu}{2} \frac{1-\nu}{2} u_{2}+\frac{1-\mu}{2} u_{3}\right)^{m}}{\sqrt{(1-\mu)\left(1-\nu^{2}\right)}} d \mu d \nu
$$

The speeds $\mu_{i}^{(m)}$ 's of (2.15) for $m>1$ are connected to $\mu_{i}^{(m)}$ 's for $m=1$, which are also given by (2.12).

Lemma 2.1. For $i=1,2,3$,

$$
\begin{aligned}
\mu_{i}^{(m)}\left(u_{1}, u_{2}, u_{3}\right)= & \frac{1}{2}\left[\lambda_{i}\left(u_{1}, u_{2}, u_{3}\right)-2\left(u_{1}+u_{2}+u_{3}\right)\right] \frac{\partial q\left(u_{1}, u_{2}, u_{3}\right)}{\partial u_{i}} \\
& +q\left(u_{1}, u_{2}, u_{3}\right) .
\end{aligned}
$$


Proof. We use (2.15), (2.16) and (2.21) to write

$$
\begin{aligned}
\mu_{1}^{(m)}\left(u_{1}, u_{2}, u_{3}\right) & =\frac{Q\left(u_{1}, u_{1}, u_{2}, u_{3}\right)}{P_{0}\left(u_{1}, u_{1}, u_{2}, u_{3}\right)} \\
& =\frac{2\left(u_{1}-u_{2}\right)\left(u_{1}-u_{3}\right)}{P_{0}\left(u_{1}, u_{1}, u_{2}, u_{3}\right)} \frac{\partial q\left(u_{1}, u_{2}, u_{3}\right)}{\partial u_{i}}+q\left(u_{1}, u_{2}, u_{3}\right) .
\end{aligned}
$$

In particular, when $m=1$, since the corresponding $q=2\left(u_{1}+u_{2}+u_{3}\right)$, we obtain

$$
\lambda_{1}\left(u_{1}, u_{2}, u_{3}\right)=\frac{4\left(u_{1}-u_{2}\right)\left(u_{1}-u_{3}\right)}{P\left(u_{1}, u_{1}, u_{2}, u_{3}\right)}+2\left(u_{1}+u_{2}+u_{3}\right) .
$$

This together with (2.26) proves formula (2.25) for $i=1$. The cases for $i=2,3$ can be shown in the same way.

Lemma 2.2. [12]

1.

$$
\begin{aligned}
& \frac{\partial \mu_{i}^{(m)}}{\partial u_{j}}= \\
& \frac{\frac{\partial \lambda_{i}}{\partial u_{j}}}{\lambda_{i}-\lambda_{j}}\left[\mu_{i}^{(m)}-\mu_{j}^{(m)}\right], \quad i, j=1,2,3 ; i \neq j .
\end{aligned}
$$

2.

$\frac{\partial}{\partial u_{i}}\left(\frac{P_{m}\left(\xi, u_{1}, u_{2}, u_{3}\right)}{\sqrt{\left(\xi-u_{1}\right)\left(\xi-u_{2}\right)\left(\xi-u_{3}\right)}}\right)=\frac{\mu_{i}^{(m)}\left(u_{1}, u_{2}, u_{3}\right)}{4^{m}(2 m+1)} \frac{\partial}{\partial u_{i}}\left(\frac{P_{0}\left(\xi, u_{1}, u_{2}, u_{3}\right)}{\sqrt{\left(\xi-u_{1}\right)\left(\xi-u_{2}\right)\left(\xi-u_{3}\right)}}\right)$

for $i=1,2,3$ and $\eta \neq u_{1}, u_{2}, u_{3}$.

The following calculations are useful in the subsequent sections.

Using formula (2.25) for $\mu_{2}$ and $\mu_{3}$ and formulae (2.12) for $\lambda_{2}$ and $\lambda_{3}$, we obtain

$$
\mu_{2}^{(m)}\left(u_{1}, u_{2}, u_{3}\right)-\mu_{3}^{(m)}\left(u_{1}, u_{2}, u_{3}\right)=\frac{2\left(u_{2}-u_{3}\right) K}{(K-E)[E-(1-s) K]} M\left(u_{1}, u_{2}, u_{3}\right),
$$

where

$$
M\left(u_{1}, u_{2}, u_{3}\right)=\left[\frac{\partial q}{\partial u_{3}}+(1-s) \frac{\partial q}{\partial u_{2}}\right] E-(1-s)\left(\frac{\partial q}{\partial u_{2}}+\frac{\partial q}{\partial u_{3}}\right) K .
$$

We then use (2.9), (2.10) and (2.22) to calculate

$$
\begin{aligned}
& \frac{\partial M\left(u_{1}, u_{2}, u_{3}\right)}{\partial u_{2}}=\frac{1}{2} \frac{p_{1}\left(u_{1}, u_{2}, u_{3}\right)}{u_{1}-u_{3}}[E-K], \\
& \frac{\partial M\left(u_{1}, u_{2}, u_{3}\right)}{\partial u_{3}}=\frac{1}{2} \frac{p_{2}\left(u_{1}, u_{2}, u_{3}\right)}{u_{1}-u_{3}}[E-(1-s) K]+\frac{3}{2} \frac{M\left(u_{1}, u_{2}, u_{3}\right)}{u_{1}-u_{3}},
\end{aligned}
$$

where

$$
\begin{aligned}
p_{1}\left(u_{1}, u_{2}, u_{3}\right) & =2\left(u_{1}-u_{2}\right) \frac{\partial}{\partial u_{2}} \operatorname{div}(q)-\operatorname{div}(q), p_{2}\left(u_{1}, u_{2}, u_{3}\right) \\
& =2\left(u_{1}-u_{3}\right) \frac{\partial}{\partial u_{3}} \operatorname{div}(q)-\operatorname{div}(q) .
\end{aligned}
$$

We next consider

$$
F\left(u_{1}, u_{2}, u_{3}\right):=\frac{\mu_{2}\left(u_{1}, u_{2}, u_{3}\right)-\mu_{3}\left(u_{1}, u_{2}, u_{3}\right)}{u_{2}-u_{3}} .
$$


Using formula (2.25) for $\mu_{2}$ and $\mu_{3}$ and formulae (2.12) for $\lambda_{2}$ and $\lambda_{3}$, we obtain

$$
\begin{aligned}
F & =-2 \frac{(1-s) K}{E-(1-s) K} \frac{\partial q}{\partial u_{2}}+2 \frac{K}{K-E} \frac{\partial q}{\partial u_{3}} \\
& =-4 \frac{s(1-s) K}{E-(1-s) K}\left(u_{1}-u_{3}\right) \frac{\partial^{2} q}{\partial u_{2} \partial u_{3}}+2\left[\frac{K}{K-E}-\frac{(1-s) K}{E-(1-s) K}\right] \frac{\partial q}{\partial u_{3}},
\end{aligned}
$$

where we have used equations (2.22) in the last equality. Finally, we use the expansions (2.5-2.6) for $K$ and $E$ to obtain

$$
F\left(u_{1}, u_{2}, u_{3}\right)=-4\left[\left(2-\frac{7}{4} s+\cdots\right)\left(u_{1}-u_{3}\right) \frac{\partial^{2} q}{\partial u_{2} \partial u_{3}}+\left(-\frac{3}{4}+O\left(s^{2}\right)\right) \frac{\partial q}{\partial u_{3}}\right] \text {. }
$$

\section{Self-similar Solutions}

In this section, we construct the self-similar solution of the Whitham equations (1.6) when $m \geq 2$ for the initial function (1.7). The $m=2$ result has already been obtained in $[\mathbf{9}]$. Even in the $m=2$ case, the key calculations presented here are different from those in $[\mathbf{9}]$.

THEOREM 3.1. (see Figure 1.) For the step-like initial data $u_{0}(x)$ of (1.7), the solution of the Whitham equations (1.6) is given by

$$
u_{1}=1, \quad x=\mu_{2}^{(m)}\left(1, u_{2}, u_{3}\right) t, \quad x=\mu_{3}^{(m)}\left(1, u_{2}, u_{3}\right) t
$$

for $\alpha t<x \leq \beta t$ and by

$$
u_{1}=1, \quad x=\mu_{2}^{(m)}\left(1, u_{2}, 0\right) t, \quad u_{3}=0
$$

for $\beta t \leq x<\gamma t$, where $\alpha=\mu_{2}^{(m)}\left(1, u^{*}, u^{*}\right), \beta=\mu_{2}^{(m)}\left(1, u^{* *}, 0\right)$ and $\gamma=\mu_{2}^{(m)}(1,1,0)=$ $q(1,1,0)=4^{m}$. Here, $u^{*}$ is uniquely determined by the equation

$$
p_{1}\left(1, u^{*}, u^{*}\right)=0,
$$

and $u^{* *}$ is uniquely given by the equation

$$
\mu_{2}^{(m)}\left(1, u^{* *}, 0\right)-\mu_{3}^{(m)}\left(1, u^{* *}, 0\right)=0 .
$$

Outside the region $\alpha t<x<4^{m} t$, the solution of the Burgers type equation (1.5) is given by

$$
u \equiv 1 \quad x \leq \alpha t
$$

and

$$
u \equiv 0 \quad x \geq 4^{m} t
$$

The boundaries $x=\alpha t$ and $x=4^{m} t$ are called the trailing and leading edges, respectively. They separate the solutions of the Whitham equations and Burgers type equations. The Whitham solution matches the Burgers type solution in the following fashion (see Figure 1.):

$$
\begin{aligned}
& u_{1}=\text { the Burgers type solution defined outside the region, } \\
& u_{2}=u_{3}
\end{aligned}
$$

at the trailing edge;

$$
\begin{aligned}
& u_{1}=u_{2}, \\
& u_{3}=\text { the Burgers type solution defined outside the region, }
\end{aligned}
$$

at the leading edge. 


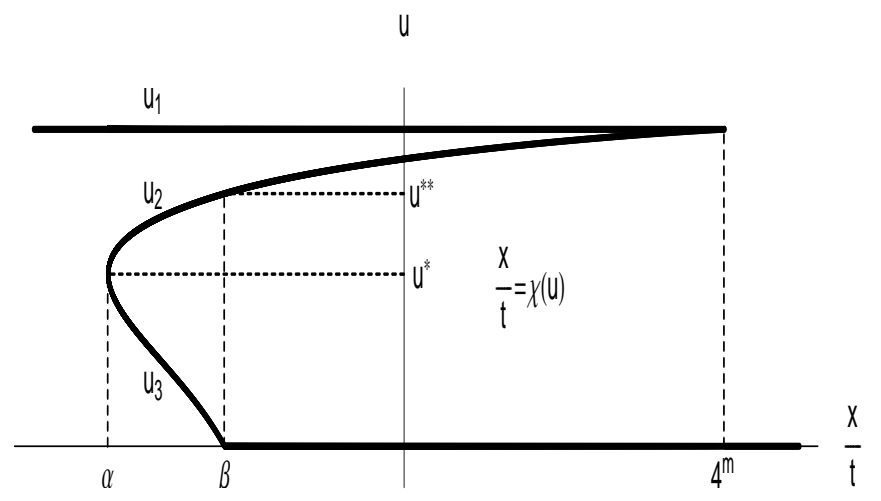

FigurE 1. Self-Similar solution of the Whitham equations for $m \geq$ 2. The curve defines the function $\chi(u)$.

The proof of Theorem 3.1 is based on a series of lemmas.

We first show that the solution defined by either formulae (3.1) or (3.2) indeed satisfies the Whitham equations $(1.6)[\mathbf{1}, \mathbf{9}, \mathbf{1 3}]$.

LEMMA 3.2. (1) The functions $u_{1}, u_{2}$ and $u_{3}$ determined by equations (3.1) give a solution of the Whitham equations (1.6) as long as $u_{2}$ and $u_{3}$ can be solved from (3.1) as functions of $x$ and $t$.

(2) The functions $u_{1}, u_{2}$ and $u_{3}$ determined by equations (3.2) give a solution of the Whitham equations (1.6) as long as $u_{2}$ can be solved from (3.2) as a function of $x$ and $t$.

Proof. (1) $u_{1}$ obviously satisfies the first equation of (1.6). To verify the second and third equations, we observe that

$$
\frac{\partial \mu_{2}^{(m)}}{\partial u_{3}}=\frac{\partial \mu_{3}^{(m)}}{\partial u_{2}}=0
$$

on the solution of (3.1). To see this, we use (2.27) to calculate

$$
\frac{\partial \mu_{2}^{(m)}}{\partial u_{3}}=\frac{\frac{\partial \lambda_{2}}{\partial u_{3}}}{\lambda_{2}-\lambda_{3}}\left(\mu_{2}^{(m)}-\mu_{3}^{(m)}\right)=0 .
$$

The second part of (3.11) can be shown in the same way.

We then calculate the partial derivatives of the second equation of (3.1) with respect to $x$ and $t$.

$$
1=\frac{\partial \mu_{2}^{(m)}}{\partial u_{2}} t u_{2 x}, \quad 0=\frac{\partial \mu_{2}^{(m)}}{\partial u_{2}} t u_{2 t}+\mu_{2}^{(m)},
$$

which give the second equation of (1.6).

The third equation of (1.6) can be verified in the same way.

(2) The second part of Lemma 3.2 can easily be proved.

We now determine the trailing edge. Eliminating $x$ and $t$ from the last two equations of (3.1) yields

$$
\mu_{2}^{(m)}\left(1, u_{2}, u_{3}\right)-\mu_{3}^{(m)}\left(1, u_{2}, u_{3}\right)=0 .
$$


Since it degenerates at $u_{2}=u_{3}$, we replace (3.12) by

$$
F\left(1, u_{2}, u_{3}\right):=\frac{\mu_{2}^{(m)}\left(1, u_{2}, u_{3}\right)-\mu_{3}^{(m)}\left(1, u_{2}, u_{3}\right)}{u_{2}-u_{3}}=0 .
$$

Here, the function $F$ is also defined in (2.34).

Therefore, at the trailing edge where $u_{2}=u_{3}$, i.e., $s=0$, equation (3.13), in view of the expansion (2.35), becomes

$$
8\left(1-u_{2}\right) \frac{\partial^{2} q\left(1, u_{2}, u_{2}\right)}{\partial u_{2} \partial u_{3}}-3 \frac{\partial q\left(1, u_{2}, u_{2}\right)}{\partial u_{3}}=0 .
$$

Since $\frac{\partial q}{\partial u_{2}}=\frac{\partial q}{\partial u_{3}}$ and $\frac{\partial^{2} q}{\partial u_{2}^{2}}=3 \frac{\partial^{2} q}{\partial u_{2} \partial u_{3}}$ on $u_{2}=u_{3}$ because of (2.22), this equation is exactly equation (3.3).

Lemma 3.3. Equation $p_{1}(1, \xi, \xi)=0$ has a simple zero, denoted by $u^{*}$, in the region $0<\xi<1$, counting multiplicities. Furthermore, $p_{1}(1, \xi, \xi)$ is positive when $\xi<u^{*}$ and negative when $\xi>u^{*}$.

Proof. We first simplify the polynomial $p_{1}$ of (2.33). In view of formula (2.24) for $q$, we use the fact that $q$ is symmetric in $u_{1}, u_{2}$ and $u_{3}$ to obtain

$$
\left.\left(\frac{\partial q\left(u_{1}, u_{2}, u_{3}\right)}{\partial u_{1}}+\frac{\partial q\left(u_{1}, u_{2}, u_{3}\right)}{\partial u_{2}}+\frac{\partial q\left(u_{1}, u_{2}, u_{3}\right)}{\partial u_{3}}\right)\right|_{u_{2}=u_{3}=\xi}=C_{m} U_{0}\left(\xi, u_{1}\right)
$$

where

$$
U_{0}\left(\xi, u_{1}\right)=\frac{1}{2 \sqrt{2}} \int_{-1}^{1} \frac{m\left(\frac{1+\mu}{2} \xi+\frac{1-\mu}{2} u_{1}\right)^{m-1}}{\sqrt{1-\mu}} d \mu .
$$

We can then write $p_{1}$ as

$$
p_{1}(1, \xi, \xi)=C_{m}\left[(1-\xi) \frac{\partial U_{0}(\xi, 1)}{\partial \xi}-U_{0}(\xi, 1)\right] .
$$

Denoting the function in the parenthesis of (3.16) by $p(\xi)$, we claim that

$$
\frac{d^{k} p(0)}{d \xi^{k}}>0, \frac{d^{k} p(1)}{d \xi^{k}}<0
$$

for $k=0,1,2, \cdots, m-2$.

Obviously,

$$
\frac{d^{k} p(\xi)}{d \xi^{k}}=(1-\xi) \frac{d^{k+1} U_{0}(\xi, 1)}{d \xi^{k+1}}-(k+1) \frac{d^{k} U_{0}(\xi, 1)}{d \xi^{k}} .
$$

Since, $\frac{d^{k} U_{0}(\xi, 1)}{d \xi^{k}}$ is a positive function, this proves the second inequality of (3.17).

To prove the first inequality of (3.17), we use formula (3.15) to calculate

$$
\left.\frac{d^{k} U_{0}(\xi, 1)}{d \xi^{k}}\right|_{\xi=0}=\frac{m(m-1) \cdots(m-k)}{2^{m+\frac{1}{2}}} \int_{-1}^{1}(1-\mu)^{m-k-\frac{3}{2}}(1+\mu)^{k} d \mu .
$$

The integral on the right can be evaluated using an iteration formula. Denote this integral by $A_{m, k}$. An integration by parts gives $A_{m, k}=\frac{2 k}{2 m-2 k-1} A_{m, k-1}$. Since $A_{m, 0}=2^{m+\frac{1}{2}} /(2 m-1)$, we thus obtain

$$
A_{m, k}=\frac{2^{m+k+\frac{1}{2}} k !}{(2 m-1)(2 m-3) \cdots(2 m-2 k-1)},
$$


which gives

$$
\left.\frac{d^{k} U_{0}(\xi, 1)}{d \xi^{k}}\right|_{\xi=0}=\frac{2^{k} k ! m(m-1) \cdots(m-k)}{(2 m-1)(2 m-3) \cdots(2 m-2 k-1)} .
$$

Therefore

$$
\begin{aligned}
& \frac{d^{k} p(0)}{d \xi^{k}}=\left.\frac{d^{k+1} U_{0}(\xi, 1)}{d \xi^{k+1}}\right|_{\xi=0}-\left.(k+1) \frac{d^{k} U_{0}(\xi, 1)}{d \xi^{k}}\right|_{\xi=0} \\
& =\frac{2^{k}(k+1) ! m(m-1) \cdots(m-k)}{(2 m-1)(2 m-3) \cdots(2 m-2 k-3)}>0 .
\end{aligned}
$$

We now use (3.17) to prove the existence and uniqueness of the zero of function $p^{(k)}(\xi), k=0,1, \cdots,(m-2)$. First, it follows from (3.17) that $p^{(k)}(\xi)$ has an odd number of zeros in $0<\xi<1$, counting multiplicities. Second, if $p^{(k)}(\xi)$ has more than one zero, it must have at least three zeros. Consequently, $p^{(k+1)}(\xi)$ will have more than one zero; so it must also have at least three zeros. Repeating this argument, we see that $p^{(m-2)}$ must have at least three zeros. This is an impossibility since $p(\xi)$ is a polynomial of degree $m-1$ because $U_{0}(\xi, 1)$ is so. Therefore, $p^{(k)}(\xi)$ has one and only one zero for $\xi \in(0,1)$ when $k=0,1, \cdots,(m-2)$. In particular, the $k=0$ case proves Lemma 3.3.

LEMma 3.4. Equation (3.13) has a unique solution satisfying $u_{2}=u_{3}$. The solution is $u_{2}=u_{3}=u^{*}$. The rest of equations (3.1) at the trailing edge are $u_{1}=1$ and $x / t=\mu_{2}^{(m)}\left(1, u^{*}, u^{*}\right)$.

Having located the trailing edge, we now solve equations (3.1) in the neighborhood of the trailing edge. We first consider equation (3.13). We use (2.35) to differentiate $F$ at the trailing edge $u_{1}=1, u_{2}=u_{3}=u^{*}$

$$
\begin{aligned}
\frac{\partial F\left(1, u^{*}, u^{*}\right)}{\partial u_{2}}=\frac{\partial F\left(1, u^{*}, u^{*}\right)}{\partial u_{3}} & =10 \frac{\partial^{2} q\left(1, u^{*}, u^{*}\right)}{\partial u_{2} \partial u_{3}}-8\left(1-u^{*}\right) \frac{\partial^{3} q\left(1, u^{*}, u^{*}\right)}{\partial u_{2}^{2} \partial u_{3}}>0 \\
& =-\frac{C_{m}}{2} \frac{\partial}{\partial \xi}\left[(1-\xi) \frac{\partial U_{0}(\xi, 1)}{\partial \xi}-U_{0}(\xi, 1)\right]_{\xi=u^{*}}>0,
\end{aligned}
$$

where in the second equality we have used (2.22), (3.14) and identities $\frac{\partial^{2} q}{\partial u_{2}^{2}}=\frac{\partial^{2} q}{\partial u_{3}^{2}}=$ $3 \frac{\partial^{2} q}{\partial u_{2} \partial u_{3}}$ on $u_{2}=u_{3}$. The inequality is a consequence of Lemma 3.3 .

Inequality (3.19) shows that equation (3.13) or equivalently (3.12) can be inverted to give $u_{2}$ as a decreasing function of $u_{3}$

$$
u_{2}=B\left(u_{3}\right)
$$

in a neighborhood of $u_{2}=u_{3}=u^{*}$.

We will extend the solution (3.20) of equation (3.12) by decreasing $u_{3}$ in the region $0<u_{3}<u^{*}<u_{2}<1$ as far as possible. We need to evaluate the derivatives $\frac{\partial\left(\mu_{2}^{(m)}-\mu_{3}^{(m)}\right)}{\partial u_{2}}$ and $\frac{\partial\left(\mu_{2}^{(m)}-\mu_{3}^{(m)}\right)}{\partial u_{3}}$ on the solution of (3.1). It follows from (2.29), (2.31) and (2.32) that

$$
\begin{aligned}
& \frac{\partial\left[\mu_{2}^{(m)}-\mu_{3}^{(m)}\right]}{\partial u_{2}}=-\frac{s K p_{1}\left(1, u_{2}, u_{3}\right)}{E-(1-s) K}, \\
& \frac{\partial\left[\mu_{2}^{(m)}-\mu_{3}^{(m)}\right]}{\partial u_{3}}=\frac{s K p_{2}\left(1, u_{2}, u_{3}\right)}{K-E},
\end{aligned}
$$


on the solution of (3.1).

We first study the two polynomials $p_{1}$ and $p_{2}$ of (2.33).

LEMma 3.5. For each $0 \leq u_{3}<1$, the polynomial $p_{1}\left(1, u_{2}, u_{3}\right)$, as a function of $u_{2}$, has only one zero in the region $0<u_{2}<1$, counting multiplicities. Furthermore, $p_{1}\left(1, u_{2}, u_{3}\right)$ is positive when $u_{2}$ is on the left of this zero and negative when $u_{2}$ is on the right.

For each $0 \leq u_{2}<1$, the polynomial $p_{2}\left(1, u_{2}, u_{3}\right)$, as a function of $u_{3}$, has only one zero in the region $0<u_{3}<1$, counting multiplicities. Furthermore, $p_{1}\left(1, u_{2}, u_{3}\right)$ is positive when $u_{3}$ is on the left of this zero and negative when $u_{3}$ is on the right.

Proof. We will prove the first part of the lemma; the second part follows from $p_{2}\left(1, u_{2}, u_{3}\right)=p_{1}\left(1, u_{3}, u_{2}\right)$.

The proof of the first part is similar to the proof of Lemma 3.3. We will go through it briefly.

We first have

$$
\frac{\partial^{k} p_{1}\left(1,0, u_{3}\right)}{\partial u_{2}^{k}}>0, \quad \frac{\partial^{k} p_{1}\left(1,1, u_{3}\right)}{\partial u_{2}^{k}}<0
$$

for $k=0,1,2, \cdots, m-2$. The second inequality immediately follows from formula (2.33) for $p_{1}$. The first inequality is derived from a formula similar to (3.18).

The rest of the proof is the same as the proof of Lemma 3.3.

We now continue to extend the solution (3.20) of equation (3.12) in the region $0<u_{3}<u^{*}<u_{2}<1$ as far as possible. When $u_{2}$ and $u_{3}$ are close to $u^{*}$, because of (3.19), we have $\frac{\partial\left(\mu_{2}^{(m)}-\mu_{3}^{(m)}\right)}{\partial u_{2}}>0$ and $\frac{\partial\left(\mu_{2}^{(m)}-\mu_{3}^{(m)}\right)}{\partial u_{3}}>0$ on the solution of (3.12). These along with (3.21) and (3.22) show that

$$
p_{1}\left(1, u_{2}, u_{3}\right)<0, p_{2}\left(1, u_{2}, u_{3}\right)>0
$$

when $u_{2}$ and $u_{3}$ are close to $u^{*}$.

LEMma 3.6. Inequalities (3.23) hold on the solution of equation (3.12) as long as $0<u_{3}<u^{*}<u_{2}<1$.

Proof. We first prove the first inequality of (3.23). In view of (2.29), the solution of equation (3.12) is also governed by an equivalent equation $M\left(1, u_{2}, u_{3}\right)=0$. For each $0<u_{3}<u^{*}$, we study the zero of the $u_{2}$-variable function $M\left(1, u_{2}, u_{3}\right)$ in the interval $u_{3}<u_{2}<1$. We obtain from formula (2.30) for $M$ that $M\left(1, u_{3}, u_{3}\right)=$ 0 and $M\left(1,1, u_{3}\right)>0$. $M\left(1, u_{2}, u_{3}\right)$ is a decreasing function of $u_{2}$ when $u_{2}$ is on the immediate right of $u_{3}$. To see this, we note that $p_{1}\left(1, u_{3}, u_{3}\right)>0$ for $u_{3}<u^{*}$ according to Lemma 3.3. This and (2.31) prove that $M$ is decreasing for $u_{2}$ on the immediate right of $u_{3}$ because $E-K<0$ for $s>0$; so $M\left(1, u_{2}, u_{3}\right)<0$ for such $u_{2}$. Therefore, $M\left(1, u_{2}, u_{3}\right)$ has a $u_{2}$-zero in the interval $u_{3}<u_{2}<1$ when $0<u_{3}<u^{*}$. Because of the uniqueness of the $u_{2}$-zero of $p\left(1, u_{2}, u_{3}\right)$ according to Lemma 3.5 , we conclude that $M\left(1, u_{2}, u_{3}\right)$ has only one zero and that this zero is on the right of the zero of $p_{1}$. Hence, the zero of $M$ is exactly given by $u_{2}=B\left(u_{3}\right)$ and $p_{1}$ is negative on the solution. This proves the first inequality of (3.23).

We now prove the second inequality of (3.23) by contradiction. Suppose it first fails at $\bar{u}_{2}$ and $\bar{u}_{3}$, where $0<\bar{u}_{3}<u^{*}<\bar{u}_{2}<1$; i.e.,

$$
p_{2}\left(1, B\left(u_{3}\right), u_{3}\right)>0 \text { when } \bar{u}_{3}<u_{3}<u^{*}, \quad p_{2}\left(1, B\left(u_{3}\right), u_{3}\right)=0 \text { when } u_{3}=\bar{u}_{3} .
$$


It then follows from (3.21) and (3.22) that solution (3.20) of equation (3.12) has a zero derivative at $u_{3}=\bar{u}_{3}$; i.e.,

$$
B^{\prime}\left(\bar{u}_{3}\right)=0 .
$$

Hence,

$\left.\frac{d}{d u_{3}} p_{2}\left(1, B\left(u_{3}\right), u_{3}\right)\right|_{u_{3}=\bar{u}_{3}}=\frac{\partial}{\partial u_{2}} p_{2}\left(1, B\left(\bar{u}_{3}\right), \bar{u}_{3}\right) B^{\prime}\left(\bar{u}_{3}\right)+\frac{\partial}{\partial u_{3}} p_{2}\left(1, B\left(\bar{u}_{3}\right), \bar{u}_{3}\right)<0$,

where the first term vanishes because of $B^{\prime}\left(\bar{u}_{3}\right)=0$ and the second term is negative according to Lemma 3.5. In view of $p_{2}\left(1, B\left(\bar{u}_{3}\right), \bar{u}_{3}\right)=0$, this implies that $p_{2}\left(1, B\left(u_{3}\right), u_{3}\right)<0$ when $u_{3}$ is on the immediately right of $\bar{u}_{3}$. That contradicts (3.24). This proves the second inequality of (3.23).

It follows from (3.21), (3.22) and Lemma 3.6 that

$$
\frac{\partial\left[\mu_{2}^{(m)}-\mu_{3}^{(m)}\right]}{\partial u_{2}}>0, \quad \frac{\partial\left[\mu_{2}^{(m)}-\mu_{3}^{(m)}\right]}{\partial u_{3}}>0
$$

on the solution of (3.12). Solution (3.20) of equation (3.12) can then be extended as a decreasing function of $u_{3}$ as long as $0<u_{3}<u^{*}<u_{2}<1$.

There are two possibilities: (1) $u_{2}$ touches 1 before or simultaneously as $u_{3}$ reaches 0 and (2) $u_{3}$ touches 0 before $u_{2}$ reaches 1 .

It follows from (2.14) and (2.25) that

$$
\mu_{2}^{(m)}\left(1,1, u_{3}\right)>\mu_{3}^{(m)}\left(1,1, u_{3}\right) \text { for } 0 \leq u_{3}<1 .
$$

This shows that (1) is impossible. Hence, $u_{3}$ will touch 0 before $u_{2}$ reaches 1 . When this happens, equation (3.12) becomes equation (3.4).

LEMMA 3.7. Equation (3.4) has a simple zero in the region $0<u_{2}<1$, counting multiplicities. Denoting the zero by $u^{* *}$, then $\mu_{2}^{(m)}\left(1, u_{2}, 0\right)-\mu_{3}^{(m)}\left(1, u_{2}, 0\right)$ is positive for $u_{2}>u^{* *}$ and negative for $u_{2}<u^{* *}$. Furthermore, $0<u^{* * *}<u^{* *}<1$ where $u^{* * *}$ is the unique zero of $p_{1}\left(1, u_{2}, 0\right)$.

Proof. We use (2.29) and (2.31) to prove the lemma. In equation (2.29), $K-E$ and $E-(1-s) K$ are all positive for $0<s<1$ in view of (2.11).

Denoting the unique zero of $p_{1}\left(1, u_{2}, 0\right)$ by $u^{* * *}$, it then follows from Lemma 3.5 that $p_{1}\left(1, u_{2}, 0\right)>0$ when $0<u_{2}<u^{* * *}$ and $p_{1}\left(1, u_{2}, 0\right)<0$ when $u^{* * *}<u_{2}<1$. Since $M\left(1, u_{2}, 0\right)$ of $(2.30)$ vanishes at $u_{2}=0$ and is positive at $u_{2}=1$ in view of (2.5-2.8), we conclude from the derivative $(2.31)$ that $M\left(1, u_{2}, 0\right)$ has a simple zero in $0<u_{2}<1$. This zero is exactly $u^{* *}$ and the rest of the theorem can be proved easily.

Having solved equation (3.12) for $u_{2}$ as a decreasing function of $u_{3}$ for $0<$ $u_{3}<u^{*}$, we turn to equations (3.1). Because of (3.11) and (3.25), the third equation of (3.1) gives $u_{3}$ as a decreasing function of $x / t$ for $\alpha \leq x / t \leq \beta$, where $\alpha=\mu_{2}^{(m)}\left(1, u^{*}, u^{*}\right)$ and $\beta=\mu_{2}^{(m)}\left(1, u^{* *}, 0\right)$. Consequently, $u_{2}$ is an increasing function of $x / t$ in the same interval.

Lemma 3.8. The last two equations of (3.1) can be inverted to give $u_{2}$ and $u_{3}$ as increasing and decreasing functions, respectively, of the self-similarity variable $x / t$ in the interval $\alpha \leq x / t \leq \beta$. 
We now turn to equations (3.2). We first use (2.9), (2.10) and (2.12) to calculate the derivative of $\mu_{2}^{(m)}$ of $(2.25)$

$$
\begin{aligned}
\frac{\partial \mu_{2}^{(m)}}{\partial u_{2}} & =\frac{1}{2}\left[\lambda_{2}-2\left(1+u_{2}+u_{3}\right)\right] \frac{\partial^{2} q}{\partial u_{2}^{2}}+\frac{1}{2} \frac{\partial \lambda_{2}}{\partial u_{2}} \frac{\partial q}{\partial u_{2}} \\
& =\frac{2\left(u_{2}-1\right) s K}{E-(1-s) K} \frac{\partial^{2} q}{\partial u_{2}^{2}}+\left[\frac{2 s K}{E-(1-s) K}+1-\frac{E^{2}-(1-s) K^{2}}{(E-(1-s) K)^{2}}\right] \frac{\partial q}{\partial u_{2}} \\
& >-\frac{2 s K}{E-(1-s) K}\left[\left(1-u_{2}\right) \frac{\partial^{2} q}{\partial u_{2}^{2}}-\frac{\partial q}{\partial u_{2}}\right],
\end{aligned}
$$

where in the inequality we have used $(E-(1-s) K)^{2}>E^{2}-(1-s) K^{2}$, which is a consequence of (2.11).

The polynomial in the parenthesis of $(3.26)$ is connected to $p_{1}\left(1, u_{2}, 0\right)$; indeed,

$$
p_{1}\left(1, u_{2}, 0\right)=\frac{2 m+1}{m}\left[\left(1-u_{2}\right) \frac{\partial^{2} q\left(1, u_{2}, 0\right)}{\partial u_{2}^{2}}-\frac{\partial q\left(1, u_{2}, 0\right)}{\partial u_{2}}\right] .
$$

This follows from the identity

$$
2 m \frac{\partial q\left(1, u_{2}, 0\right)}{\partial u_{3}}=\left(1-u_{2}\right) \frac{\partial q\left(1, u_{2}, 0\right)}{\partial u_{2}}+m q\left(1, u_{2}, 0\right) .
$$

To see this, taking the derivative of (3.28) and using formula (2.33) for $p_{1}$ and equations (2.22) for $q$ yield (3.27).

To prove (3.28), we use the integral formula (2.24) for $q$ to calculate both sides of the identity. The left equals

$$
\begin{aligned}
& \frac{C_{m} m^{2}}{4^{m-1} 2^{3 / 2} \pi}\left[\int_{-1}^{1}(1+\mu)^{m-1}(1-\mu)^{1 / 2} d \mu\right] \\
& {\left[\int_{-1}^{1}\left((1+\nu)+(1-\nu) u_{2}\right)^{m-1}\left(1-\nu^{2}\right)^{-1 / 2} d \nu\right] .}
\end{aligned}
$$

The right is

$$
\begin{aligned}
& \frac{C_{m} m}{4^{m-1} 2^{5 / 2} \pi}\left[\int_{-1}^{1}(1+\mu)^{m}(1-\mu)^{-1 / 2} d \mu\right] \\
& {\left[\int_{-1}^{1}\left((1+\nu)+(1-\nu) u_{2}\right)^{m-1}\left(1-\nu^{2}\right)^{-1 / 2} d \nu\right] .}
\end{aligned}
$$

Both sides are equal in view of an easy identity

$$
m \int_{-1}^{1}(1+\mu)^{m-1}(1-\mu)^{1 / 2} d \mu=\frac{1}{2} \int_{-1}^{1}(1+\mu)^{m}(1-\mu)^{-1 / 2} d \mu .
$$

We have therefore proved identity (3.28).

By Lemma 3.5, $p_{1}\left(1, u_{2}, 0\right)$ is negative for $u_{2}>u^{* * *}$, where $u^{* * *}$ is the unique zero of $p_{1}$. Since $u^{* * *}<u^{* *}$ according to Lemma 3.7, we conclude from (3.26) and (3.27) that $\frac{\partial \mu_{2}^{(m)}}{\partial u_{2}}>0$ on the solution of (3.2) when $u_{2}>u^{* *}$. Hence, the second equation of (3.2) can be solved for $u_{2}$ as an increasing function of $x / t$ as long as $u^{* *}<u_{2}<1$. When $u_{2}$ reaches 1 , we have

$$
x / t=\gamma=\mu_{2}^{(m)}(1,1,0) .
$$

We have therefore proved the following result. 
LEMma 3.9. The second equation of (3.2) can be inverted to give $u_{2}$ as an increasing function of $x / t$ in the interval $\beta \leq x / t \leq \gamma$.

We are ready to conclude the proof of Theorem 3.1.

The Burgers type solutions (3.5) and (3.6) are trivial.

According to Lemma 3.8, the last two equations of (3.1) determine $u_{2}$ and $u_{3}$ as functions of $x / t$ in the region $\alpha \leq x / t \leq \beta$. By the first part of Lemma 3.2, the resulting $u_{1}, u_{2}$ and $u_{3}$ satisfy the Whitham equations (1.6). Furthermore, the boundary conditions (3.7) and (3.8) are satisfied at the trailing edge $x=\alpha t$.

Similarly, by Lemma 3.9, the second equation of (3.2) determines $u_{2}$ as a function of $x / t$ in the region $\beta \leq x / t \leq \gamma=4^{m}$. It then follows from the second part of Lemma 3.2 that $u_{1}, u_{2}$ and $u_{3}$ of (3.2) satisfy the Whitham equations (1.6). They also satisfy the boundary conditions (3.9) and (3.10) at the leading edge $x=\gamma t$.

We have therefore completed the proof of Theorem 3.1.

\section{The Minimization Problem}

The zero dispersion limit of the solution of the higher order KdV equation (1.4) with step-like initial function (1.7) is also determined by a minimization problem with constraints $[\mathbf{5}, \mathbf{6}, \mathbf{1 4}]$

$$
\begin{aligned}
& \underset{\left\{\psi \geq 0, \psi \in L^{1}\right\}}{\operatorname{Minimize}}\left\{-\frac{1}{2 \pi} \int_{0}^{1} \int_{0}^{1} \log \left|\frac{\eta-\mu}{\eta+\mu}\right| \psi(\eta) \psi(\mu) d \eta d \mu\right. \\
& \left.+\int_{0}^{1}\left[\eta x-4^{m} \eta^{2 m+1} t\right] \psi(\eta) d \eta\right\} .
\end{aligned}
$$

In this section, we will use the self-similar solution of Section 3 to construct the minimizer for $m \geq 2$. The $m=2$ result has already been obtained in [9]. Even in the $m=2$ case, the key calculations presented here are different from those in [9].

We first define a linear operator

$$
L \psi(\eta)=\frac{1}{2 \pi} \int_{0}^{1} \log \left(\frac{\eta-\mu}{\eta+\mu}\right)^{2} \psi(\mu) d \mu .
$$

The variational conditions are

$$
\begin{array}{cc}
L \psi=x \eta-4^{m} t \eta^{2 m+1} & \text { where } \psi>0, \\
L \psi \leq x \eta-4^{m} t \eta^{2 m+1} & \text { where } \psi=0 .
\end{array}
$$

The constraint for the minimization problem is

$$
\psi \geq 0 \text {. }
$$

The minimizer of (4.1) is given explicitly:

TheOREM 4.1. The minimizer of the variational problem (4.1) is as follows:

(1) For $x \leq \alpha t$,

$$
\psi(\eta)=\frac{-x \eta+4^{m}(2 m+1) t \eta \frac{P_{m}\left(\eta^{2}, 1, u^{*}, u^{*}\right)}{\eta^{2}-u^{*}}}{\sqrt{1-\eta^{2}}} .
$$


(2) For $\alpha t<x<\beta t$,

$$
\psi(\eta)=\left\{\begin{array}{cc}
-\frac{-x \eta P_{0}\left(\eta^{2}, 1, u_{2}, u_{3}\right)+4^{m}(2 m+1) t \eta P_{m}\left(\eta^{2}, 1, u_{2}, u_{3}\right)}{\sqrt{\left(1-\eta^{2}\right)\left(u_{2}-\eta^{2}\right)\left(u_{3}-\eta^{2}\right)}} & 0<\eta<\sqrt{u_{3}} \\
0 & \sqrt{u_{3}}<\eta<\sqrt{u_{2}} \\
\frac{-x \eta P_{0}\left(\eta^{2}, 1, u_{2}, u_{3}\right)+4^{m}(2 m+1) t \eta P_{m}\left(\eta^{2}, 1, u_{2}, u_{3}\right)}{\sqrt{\left(1-\eta^{2}\right)\left(\eta^{2}-u_{2}\right)\left(\eta^{2}-u_{3}\right)}} & \sqrt{u_{2}}<\eta<1,
\end{array}\right.
$$

where $P_{0}$ and $P_{m}$ are defined in (2.1) and $u_{2}$ and $u_{3}$ are determined by equations (3.1).

(3) For $\beta t<x<4^{m} t$,

$$
\psi(\eta)=\left\{\begin{array}{cc}
0 & 0<\eta<\sqrt{u_{2}} \\
\frac{-x P_{0}\left(\eta^{2}, 1, u_{2}, 0\right)+4^{m}(2 m+1) t P_{m}\left(\eta^{2}, 1, u_{2}, 0\right)}{\sqrt{\left(1-\eta^{2}\right)\left(\eta^{2}-u_{2}\right)}} & \sqrt{u_{2}}<\eta<1
\end{array}\right.
$$

where $u_{2}$ is determined by (3.2).

(4) For $x \geq 4^{m} t$,

$$
\psi(\eta) \equiv 0
$$

Proof. We extend the function $\psi$ defined on $[0,1]$ to the entire real line by setting $\psi(\eta)=0$ for $\eta>1$ and taking $\psi$ to be odd. In this way, the operator $L$ is connected to the Hilbert transform $H$ on the real line [5]:

$$
L \psi(\eta)=\int_{0}^{\eta} H \psi(\mu) d \mu \quad \text { where } H \psi(\eta)=\frac{1}{\pi} P . V . \int_{-\infty}^{+\infty} \frac{\psi(\mu)}{\eta-\mu} d \mu .
$$

We verify case (4) first. Clearly $\psi(\eta)=0$ satisfies the constraint (4.4). We now check the variational conditions (4.2-4.3). Since $\psi=0$,

$$
L \psi=0 \leq x \eta-4^{m} t \eta^{2 m+1},
$$

where the inequality follows from $x \geq 4^{m} t$ and $0 \leq \eta \leq 1$. Hence, variational conditions (4.2-4.3) are satisfied.

Next we consider case (1). We write $\psi(\eta)$ as the real part of $g_{1}(\eta)$ for real $\eta$, where

$$
g_{1}=\sqrt{-1}\left(x-4^{m}(2 m+1) t \eta^{2 m}\right)+\frac{\sqrt{-1}\left[-x \eta+4^{m}(2 m+1) t \eta \frac{P_{m}\left(\eta^{2}, 1, u^{*}, u^{*}\right)}{\eta^{2}-u^{*}}\right]}{\sqrt{\eta^{2}-1}} .
$$

The function $g_{1}$ is analytic in the upper half complex plane $\operatorname{Im}(\eta)>0$ and $g_{1}(\eta) \approx$ $O\left(1 / \eta^{2}\right)$ for large $|\eta|$ in view of the expansion (2.1) for $P_{m}$. Hence, $H \psi(\eta)=$ $\operatorname{Im}\left[g_{1}(\eta)\right]=x-4^{m}(2 m+1) t \eta^{2 m}$ on $0 \leq \eta \leq 1$, where $H$ is the Hilbert transform [5]. We then have for $0 \leq \eta \leq 1$

$$
L \psi(\eta)=\int_{0}^{\eta} H \eta(\mu) d \mu=x \eta-4^{m} t \eta^{2 m+1},
$$

which shows that the variational conditions (4.2) and (4.3) are satisfied.

To prove (4.4), we first claim that

$$
-\alpha+4^{m}(2 m+1) \frac{P_{m}\left(\eta^{2}, 1, u^{*}, u^{*}\right)}{\eta^{2}-u^{*}} \geq 0,
$$


for $0 \leq \eta \leq 1$. To see this, we use (2.13) and (2.25) to calculate $\alpha=\mu_{2}^{(m)}\left(1, u^{*}, u^{*}\right)$ and (2.16) and (2.21) to evaluate $P_{m}\left(1, u^{*}, u^{*}\right)$. The left hand side of (4.5) equals

$$
\begin{aligned}
& 2\left(\eta^{2}-1\right)\left(\eta^{2}-u^{*}\right) \Phi\left(\eta^{2}, 1, u^{*}, u^{*}\right)+2\left(\eta^{2}-u^{*}\right) \\
& {\left[\frac{\partial q\left(1, u^{*}, u^{*}\right)}{\partial u_{1}}+\frac{\partial q\left(1, u^{*}, u^{*}\right)}{\partial u_{2}}+\frac{\partial q\left(1, u^{*}, u^{*}\right)}{\partial u_{3}}\right]} \\
& =2\left(\eta^{2}-1\right)\left[C_{m} U\left(\eta^{2}, 1\right)-C_{m} U\left(u^{*}, 1\right)\right]+2\left(\eta^{2}-u^{*}\right) C_{m} U\left(u^{*}, 1\right) \\
& =2\left[\left(1-u^{*}\right) C_{m} U\left(u^{*}, 1\right)-\left(1-\eta^{2}\right) C_{m} U\left(\eta^{2}, 1\right)\right],
\end{aligned}
$$

where in the first equality we have used (3.14) and the identity (cf. (3.28) of [2])

$$
\Phi\left(\eta^{2}, 1, u^{*}, u^{*}\right)=\frac{C_{m} U\left(\eta^{2}, 1\right)-C_{m} U\left(u^{*}, 1\right)}{\eta^{2}-u^{*}} .
$$

In view of (3.16), we have

$$
\frac{d}{d\left(\eta^{2}\right)}\left[\left(1-\eta^{2}\right) C_{m} U\left(\eta^{2}, 1\right)\right]=C_{m}\left[\left(1-\eta^{2}\right) \frac{\partial U\left(\eta^{2}, 1\right)}{\partial \eta^{2}}-U\left(\eta^{2}, 1\right)\right]=p_{1}\left(1, \eta^{2}, \eta^{2}\right) .
$$

This derivative is positive when $\eta^{2}<u^{*}$ and negative when $\eta^{2}>u^{*}$ because $p_{1}$ is so according to Lemma 3.3. Therefore, $\left(1-\eta^{2}\right) C_{m} U\left(\eta^{2}, 1\right)$ has a maximum at $\eta^{2}=u^{*}$; i.e., $\left(1-\eta^{2}\right) C_{m} U\left(\eta^{2}, 1\right) \leq\left(1-u^{*}\right) C_{m} U\left(u^{*}, 1\right)$ for $0 \leq \eta^{2} \leq 1$. This together with (4.6) proves (4.5).

It follows from $x \leq \alpha t$ and inequality (4.5) that $\psi \geq 0$. Hence, the constraint (4.4) is verified.

We now turn to case (2). By Lemma 3.5, the last two equations of (3.1) determine $u_{2}$ and $u_{3}$ as functions of the self-similarity variable $x / t$ in the interval $\alpha \leq x / t \leq \beta$.

We write $\psi=\operatorname{Re}\left(g_{2}\right)$ for real $\eta$, where

$$
\begin{aligned}
& g_{2}=\sqrt{-1}\left(x-4^{m}(2 m+1) t \eta^{2 m}\right)+ \\
& \frac{\sqrt{-1}\left[-x \eta P_{0}\left(\eta^{2}, 1, u_{2}, u_{3}\right)+4^{m}(2 m+1) t \eta P_{m}\left(\eta^{2}, 1, u_{2}, u_{3}\right)\right]}{\sqrt{\left.\eta^{2}-1\right)\left(\eta^{2}-u_{2}\right)\left(\eta^{2}-u_{3}\right)}} .
\end{aligned}
$$

The function $g_{2}$ is analytic in $\operatorname{Im}(\eta)>0$ and $g_{2}(\eta) \approx O\left(1 / \eta^{2}\right)$ for large $|\eta|$ in view of the asymptotics $(2.2)$ for $P_{0}$ and $P_{m}$. Hence, taking the imaginary part of $g_{2}$ yields

$$
H \psi(\eta)=\left\{\begin{array}{cc}
x-4^{m}(2 m+1) t \eta^{2 m} & 0<\eta<\sqrt{u_{3}} \\
x-4^{m}(2 m+1) t \eta^{2 m} & \\
-\frac{\left[-x P_{0}\left(\eta^{2}, 1, u_{2}, 0\right)+4^{m}(2 m+1) t P_{m}\left(\eta^{2}, 1, u_{2}, 0\right)\right] \eta}{\sqrt{\left(1-\eta^{2}\right)\left(u_{2}-\eta^{2}\right)\left(\eta^{2}-u_{3}\right)}} & \sqrt{u_{3}}<\eta<\sqrt{u_{2}} \\
x-4^{m}(2 m+1) t \eta^{2 m} & \sqrt{u_{2}}<\eta<1 .
\end{array}\right.
$$

We then have

$$
L \psi(\eta)=\left\{\begin{array}{cc}
x \eta-4^{m} t \eta^{2 m+1} & 0<\eta<\sqrt{u_{3}} \\
x \eta-4^{m} t \eta^{2 m+1} & \\
-\int_{\sqrt{u_{3}}}^{\eta} \frac{\left[-x P_{0}+4^{m}(2 m+1) t P_{m}\right] \mu}{\sqrt{\left(1-\mu^{2}\right)\left(u_{2}-\mu^{2}\right)\left(\mu^{2}-u_{3}\right)}} d \mu & \sqrt{u_{3}}<\eta<\sqrt{u_{2}} \\
x \eta-4^{m} t \eta^{2 m+1} & \sqrt{u_{2}}<\eta<1,
\end{array}\right.
$$

where we have used

$$
\int_{\sqrt{u_{3}}}^{\sqrt{u_{2}}} \frac{\left[-x P_{0}+4^{m}(2 m+1) t P_{m}\right] \mu}{\sqrt{\left(1-\mu^{2}\right)\left(u_{2}-\mu^{2}\right)\left(\mu^{2}-u_{3}\right)}} d \mu=0
$$


which is a consequence of (2.3) for $P_{0}$ and $P_{m}$.

To verify (4.4), we derive an integral formula for $\psi$. We use (2.28) and (3.1) to calculate

$$
\psi_{x}(\eta)=\left\{\begin{array}{cc}
\frac{\eta P_{0}\left(\eta^{2}, 1, u_{2}(x, t), u_{3}(x, t)\right)}{\sqrt{\left(1-\eta^{2}\right)\left(u_{2}(x, t)-\eta^{2}\right)\left(u_{3}(x, t)-\eta^{2}\right)}} & 0<\eta<\sqrt{u_{3}(x, t)} \\
0 & \sqrt{u_{3}(x, t)}<\eta<\sqrt{u_{2}(x, t)} \\
\frac{-\eta P_{0}\left(\eta^{2}, 1, u_{2}(x, t), 0\right)}{\sqrt{\left(1-\eta^{2}\right)\left(\eta^{2}-u_{2}(x, t)\right)\left(\eta^{2}-u_{3}(x, t)\right)}} & \sqrt{u_{2}(x, t)}<\eta<1 .
\end{array}\right.
$$

Integrating yields

$$
\psi(\eta)=\left\{\begin{array}{cc}
-\int_{x}^{\chi\left(\eta^{2}\right) t} \frac{\eta P_{0}\left(\eta^{2}, 1, u_{2}(y, t), u_{3}(y, t)\right)}{\sqrt{\left(1-\eta^{2}\right)\left(u_{2}(y, t)-\eta^{2}\right)\left(u_{3}(y, t)-\eta^{2}\right)}} d y & 0<\eta<\sqrt{u_{3}(x, t)} \\
0 & \sqrt{u_{3}(x, t)}<\eta<\sqrt{u_{2}(x, t)} \\
\int_{x}^{\chi\left(\eta^{2}\right) t} \frac{\eta P_{0}\left(\eta^{2}, 1, u_{2}(y, t), 0\right)}{\sqrt{\left(1-\eta^{2}\right)\left(\eta^{2}-u_{2}(y, t)\right)\left(\eta^{2}-u_{3}(y, t)\right)}} d y & \sqrt{u_{2}(x, t)}<\eta<1,
\end{array}\right.
$$

where $\chi\left(\eta^{2}\right)$ is defined in Figure 1. The polynomial $P_{0}$ of $(2.1)$ is linear in $\eta^{2}$ and has a zero for $u_{3}<\eta^{2}<u_{2}$ because of (2.3). $P_{0}$ must be positive for $\eta^{2}>u_{2}$ and negative for $\eta^{2}<u_{3}$. This combined with (4.8) proves $\psi \geq 0$; so (4.4) is verified.

We now continue to verify the variational conditions (4.2) and (4.3). Again, we use (2.28) and (3.1) to calculate

$$
\begin{gathered}
\frac{\partial}{\partial x}\left(\frac{\left[-x P_{0}\left(\mu^{2}, 1, u_{2}(x, t), u_{3}(x, t)\right)+4^{m}(2 m+1) t P_{m}\left(\mu^{2}, 1, u_{2}(x, t), u_{3}(x, t)\right)\right] \mu}{\sqrt{\left(1-\mu^{2}\right)\left(u_{2}(x, t)-\mu^{2}\right)\left(\mu^{2}-u_{3}^{2}(x, t)\right)}}\right) \\
=\frac{-\mu P_{0}\left(\mu^{2}, 1, u_{2}(x, t), u_{3}(x, t)\right)}{\sqrt{\left(1-\mu^{2}\right)\left(u_{2}(x, t)-\mu^{2}\right)\left(\mu^{2}-u_{3}^{2}(y, t)\right)}} .
\end{gathered}
$$

Integrating yields

$$
\begin{gathered}
\frac{\left[-x P_{0}\left(\mu^{2}, 1, u_{2}(x, t), u_{3}(x, t)\right)+4^{m}(2 m+1) t P_{m}\left(\mu^{2}, 1, u_{2}(x, t), u_{3}(x, t)\right)\right] \mu}{\sqrt{\left(1-\mu^{2}\right)\left(u_{2}(x, t)-\mu^{2}\right)\left(\mu^{2}-u_{3}^{2}(x, t)\right)}} \\
=-\int_{\chi\left(\mu^{2}\right) t}^{x} \frac{\mu P_{0}\left(\mu^{2}, 1, u_{2}(y, t), u_{3}(y, t)\right)}{\sqrt{\left(1-\mu^{2}\right)\left(u_{2}(y, t)-\mu^{2}\right)\left(\mu^{2}-u_{3}^{2}(y, t)\right)}} d y
\end{gathered}
$$

for $\sqrt{u_{3}(x, t)}<\mu<\sqrt{u_{2}(x, t)}$. The single integral in (4.7) can then be written as a double integral. After interchanging integrals and using (2.3) for $P_{0}$, the double integral is simplified as

$$
-\int_{\chi\left(\eta^{2}\right) t}^{x} \int_{\sqrt{u_{3}(y, t)}}^{\eta} \frac{\mu P_{0}\left(\mu^{2}, 1, u_{2}(y, t), u_{3}(y, t)\right)}{\sqrt{\left(1-\mu^{2}\right)\left(u_{2}(y, t)-\mu^{2}\right)\left(\mu^{2}-u_{3}^{2}(y, t)\right)}} d \mu d y .
$$

The polynomial $P_{0}$ is linear in $\mu^{2}$ and has a zero for $u_{3}(y, t)<\mu^{2}<u_{2}(y, t)$. In view of (2.3) for $P_{0}$, we must have

$$
\int_{\sqrt{u_{3}(y, t)}}^{\eta} \frac{\mu P_{0}\left(\mu^{2}, 1, u_{2}(y, t), u_{3}(y, t)\right)}{\sqrt{\left(1-\mu^{2}\right)\left(u_{2}(y, t)-\mu^{2}\right)\left(\mu^{2}-u_{3}^{2}(y, t)\right)}} d \mu<0
$$

for $\sqrt{u_{3}(y, t)}<\eta<\sqrt{u_{2}(y, t)}$. Hence, the integral in (4.7) is positive and this verifies the variational conditions (4.2) and (4.3).

We finally consider case (3). By Lemma 3.6, the second equation of (3.2) determines $u_{2}$ as an increasing function of $x / t$ in the interval $\beta \leq x / t \leq 4^{m}$. 
We write $\psi=\operatorname{Re}\left(g_{3}\right)$ for real $\eta$, where

$$
\begin{aligned}
& g_{3}=\sqrt{-1}\left(x-4^{m}(2 m+1) t \eta^{2 m}\right)+ \\
& \frac{\sqrt{-1}\left[-x P_{0}\left(\eta^{2}, 1, u_{2}, 0\right)+4^{m}(2 m+1) t P_{m}\left(\eta^{2}, 1, u_{2}, 0\right)\right]}{\sqrt{\left(\eta^{2}-1\right)\left(\eta^{2}-u_{2}\right)}} .
\end{aligned}
$$

The function $g_{3}$ is analytic in $\operatorname{Im}(\eta)>0$ and $g_{3}(\eta) \approx O\left(1 / \eta^{2}\right)$ for large $|\eta|$ in view of the asymptotics (2.2) for $P_{0}$ and $P_{m}$. Hence, taking the imaginary part of $g_{3}$ yields

$$
H \psi(\eta)=\left\{\begin{array}{cc}
x-4^{m}(2 m+1) t \eta^{2 m} & \\
-\frac{-x P_{0}\left(\eta^{2}, 1, u_{2}, 0\right)+4^{m}(2 m+1) P_{m}\left(\eta^{2}, 1, u_{2}, 0\right)}{\sqrt{\left(1-\eta^{2}\right)\left(u_{2}-\eta^{2}\right)}} & 0<\eta<\sqrt{u_{2}} \\
x-4^{m}(2 m+1) t \eta^{2 m} & \sqrt{u_{2}}<\eta<1 .
\end{array}\right.
$$

We then have

$$
L \psi(\eta)=\left\{\begin{array}{cc}
x \eta-4^{m} t \eta^{2 m+1}-\int_{0}^{\eta} \frac{-x P_{0}+4^{m}(2 m+1) t P_{m}}{\sqrt{\left(1-\mu^{2}\right)\left(u_{2}-\mu^{2}\right)}} d \mu & 0<\eta<\sqrt{u_{2}} \\
x \eta-4^{m} t \eta^{2 m+1} & \sqrt{u_{2}}<\eta<1,
\end{array}\right.
$$

where we have used

$$
\int_{0}^{\sqrt{u_{2}}} \frac{-x P_{0}\left(\mu^{2}, 1, u_{2}, 0\right)+4^{m}(2 m+1) t P_{m}\left(\mu^{2}, 1, u_{2}, 0\right)}{\sqrt{\left(1-\mu^{2}\right)\left(u_{2}-\mu^{2}\right)}} d \mu=0,
$$

which is a consequence of (2.3) for $P_{0}$ and $P_{m}$.

The variational conditions (4.2-4.3) and the constraint (4.4) can be verified using the method for case (2).

Acknowledgments. We thank Tamara Grava for some of the ideas leading to the proof of Lemma 3.3. V.P. was supported in part by NSF Grant DMS-0135308. F.-R. T. was supported in part by NSF Grant DMS-0404931.

\section{References}

1. B.A. Dubrovin and S.P. Novikov, "Hydrodynamics of Weakly Deformed Soliton Lattices. Differential Geometry and Hamiltonian Theory", Russian Math. Surveys 44:6 (1989), 35-124.

2. T. Grava and F.R. Tian, "The Generation, Propagation and Extinction of Multiphases in the KdV Zero Dispersion Limit", Comm. Pure Appl. Math. 55(2002), 1569-1639.

3. A.V. Gurevich and L.P. Pitaevskii, "Non-stationary Structure of a Collisionless Shock Wave", Soviet Phys. JETP 38 (1974), 291-297.

4. I.M. Krichever, "The Method of Averaging for Two-dimensional 'Integrable' Equations", Functional Anal. App. 22 (1988), 200-213.

5. P.D. Lax and C.D. Levermore, "The Small Dispersion Limit for the Korteweg-de Vries Equation I, II, and III", Comm. Pure Appl. Math. 36 (1983), 253-290, 571-593, 809-830.

6. P.D. Lax, C.D. Levermore and S. Venakides, "The Generation and Propagation of Oscillations in Dispersive IVPs and Their Limiting Behavior" in Important Developments in Soliton Theory 1980-1990, T. Fokas and V.E. Zakharov eds., Springer-Verlag, Berlin (1992).

7. P.G. LeFloch, Hyperbolic Systems of Conservation Laws, Lectures in Mathematics, Birkhauser, 2002.

8. C.D. Levermore, "The Hyperbolic Nature of the Zero Dispersion KdV Limit", Comm. P.D.E. 13 (1988), 495-514.

9. V. Pierce and F.R. Tian, "Self-Similar Solutions of the Non-Strictly Hyperbolic Whitham Equations", Comm. Math. Sci. 4(2006), 799-822.

10. F.R. Tian, "Oscillations of the Zero Dispersion Limit of the Korteweg-de Vries Equation", Comm. Pure Appl. Math. 46 (1993), 1093-1129. 
11. F.R. Tian, "On the Initial Value Problem of the Whitham Averaged System", in Singular Limits of Dispersive Waves, N. Ercolani, I. Gabitov, D. Levermore and D. Serre eds., NATO ARW series, Series B: Physics Vol. 320, Plenum, New York (1994), 135-141.

12. F.R. Tian, "The Whitham Type Equations and Linear Overdetermined Systems of EulerPoisson-Darboux Type", Duke Math. Jour. 74 (1994), 203-221.

13. S.P. Tsarev, "Poisson Brackets and One-dimensional Hamiltonian Systems of Hydrodynamic Type", Soviet Math. Dokl. 31 (1985), 488-491.

14. S. Venakides, "The Zero Dispersion Limit of the KdV Equation with Nontrivial Reflection Coefficient", Comm. Pure Appl. Math. 38 (1985), 125-155.

15. G.B. Whitham, "Non-linear Dispersive Waves", Proc. Royal Soc. London Ser. A 139 (1965), 283-291.

Department of Mathematics, Ohio State University, 231 W. 18th Avenue, Columbus, $\mathrm{OH} 43210$

E-mail address: vpierce@math.ohio-state.edu

Department of Mathematics, Ohio State University, 231 W. 18th Avenue, Columbus, OH 43210

E-mail address: tian@math.ohio-state.edu 